\title{
Finite Element Modeling and Experimental Validation of a z-Type Self-Expanding Endovascular Stent
}

\author{
Joel Scott ${ }^{1}$, Darrel Doman ${ }^{1}$, and Clifton Johnston ${ }^{1}$ \\ ${ }^{1}$ Dalhousie University
}

September 8, 2021

\begin{abstract}
Stent migration due to haemodynamic drag remains the primary cause of type I endoleak, potentially leading to aneurysm rupture. The prevalence of migration and endoleak can be partially attributed to deficiencies in stent-graft radial spring design and a lack in understanding of the mechanical properties of endovascular stents. A converged finite element model of a custom radial extensometer was developed, fit, and validated using experimental results for bare stent wire ("uncovered") with outer diameter of $12 \mathrm{~mm}$ stent. During stent constriction to $50 \%$ of the original cross- sectional area, a comparison of experimental and modeled results produced an $\mathrm{r} 2$ value of 0.946 , a standard error of $0.099 \mathrm{~N}$, and a mean percent error of $1.69 \%$. This validated finite element model can be used to analyze the mechanisms responsible for radial force generation in 316L stainless steel self-expanding endovascular stents, as well as to evaluate new stent designs.
\end{abstract}

\section{Hosted file}

Revised Manuscript, Clean Copy.pdf available at https://authorea.com/users/433112/articles/ 536552-finite-element-modeling-and-experimental-validation-of-a-z-type-self-expandingendovascular-stent 


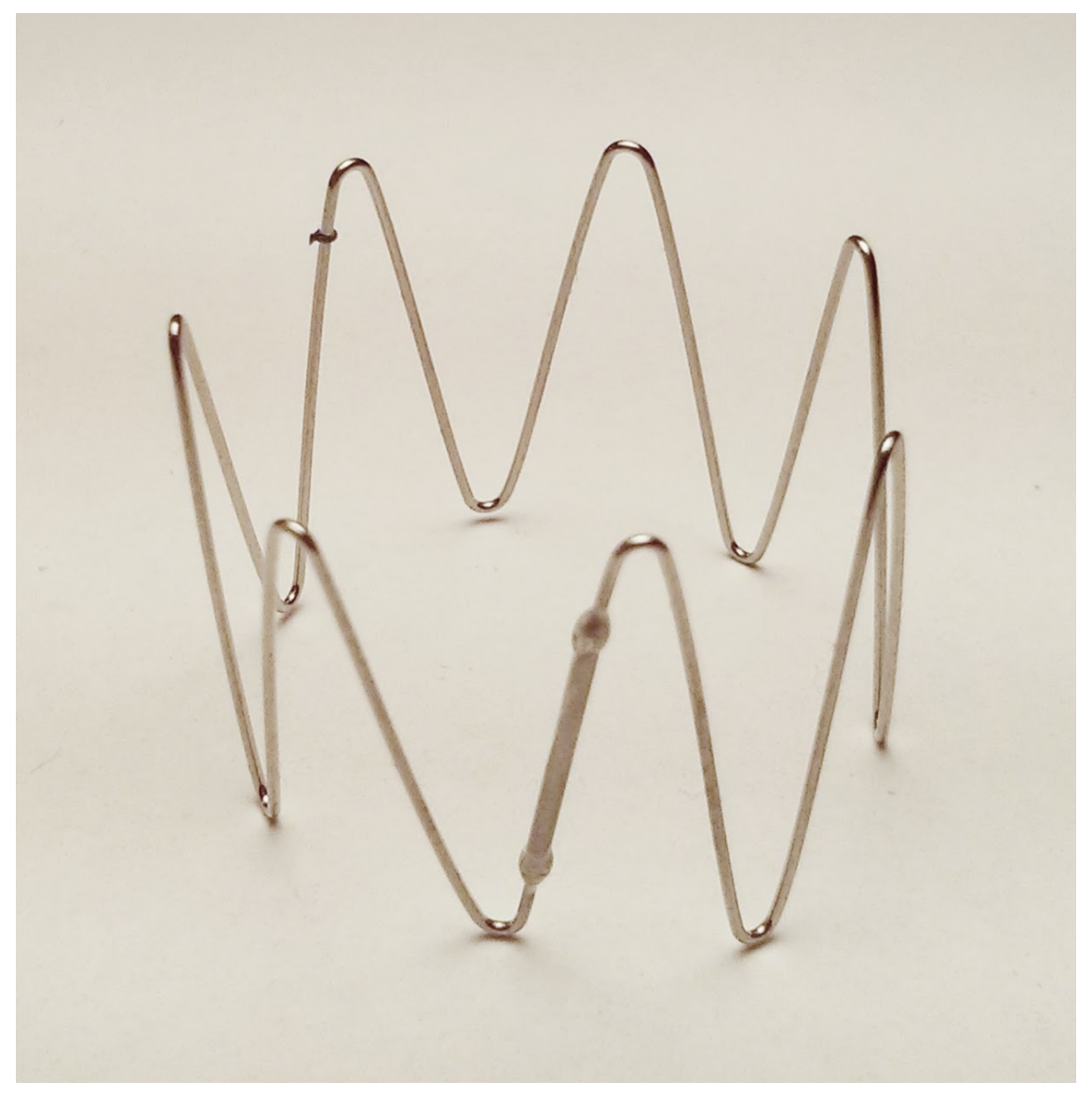



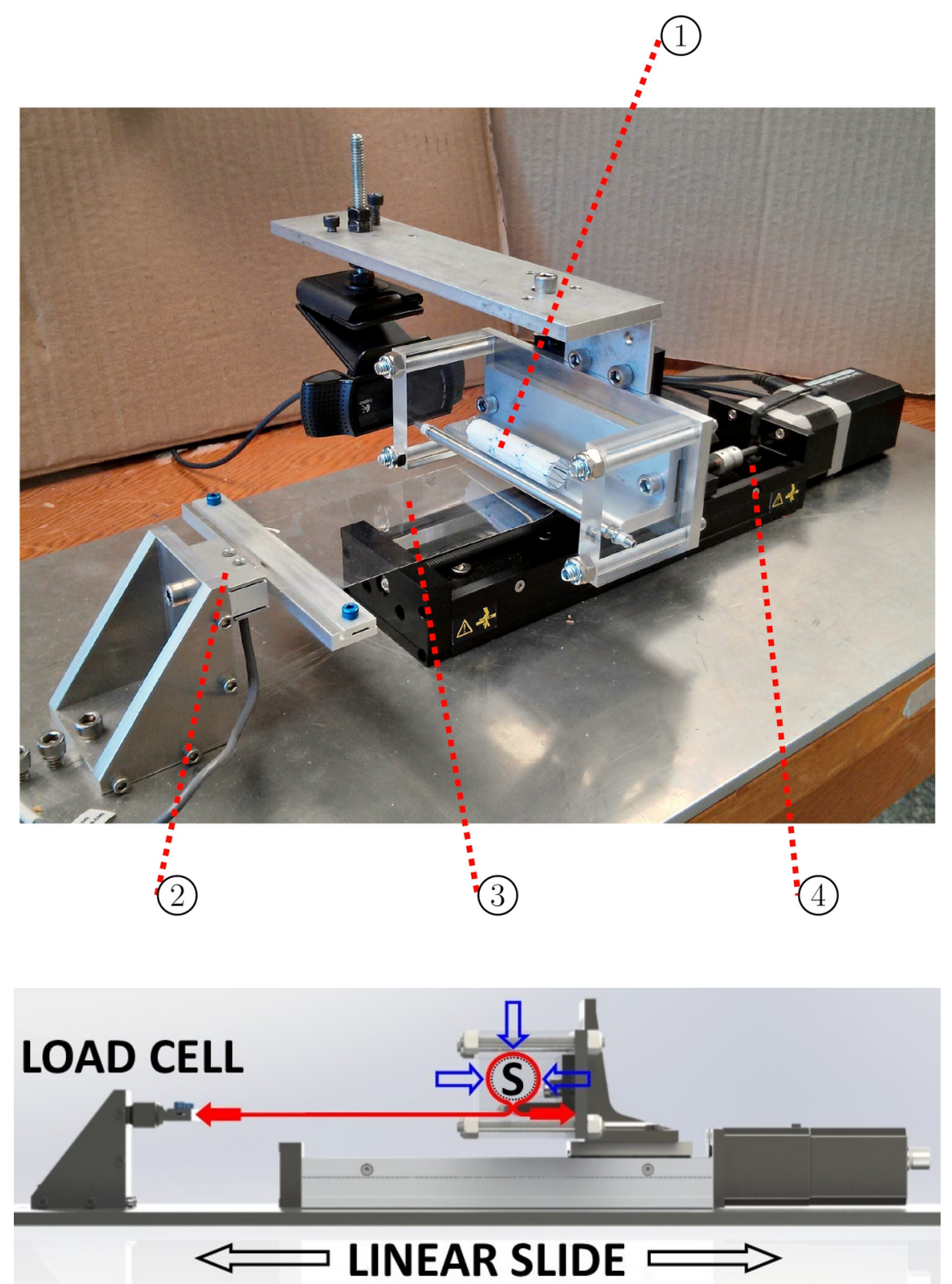


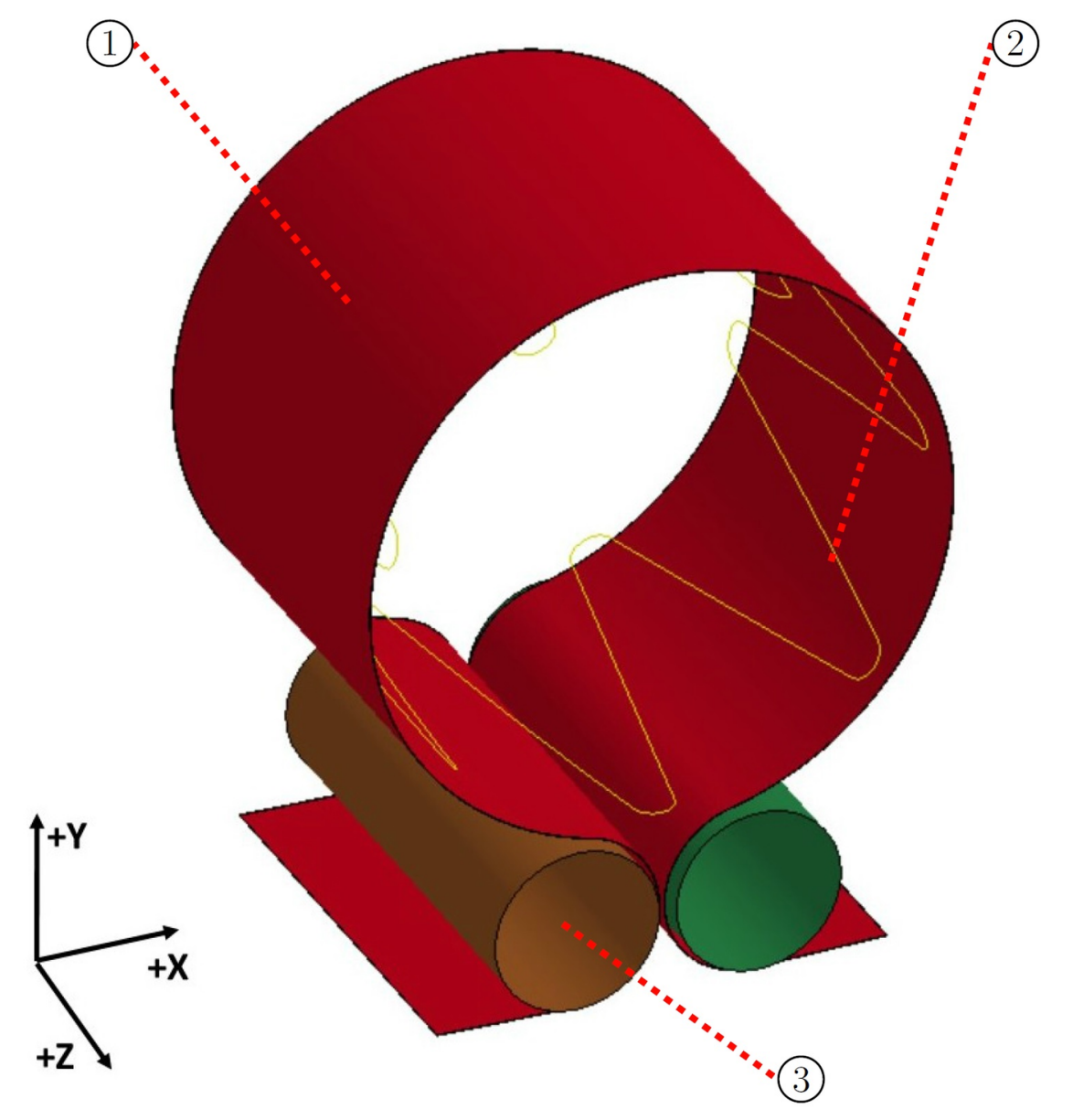




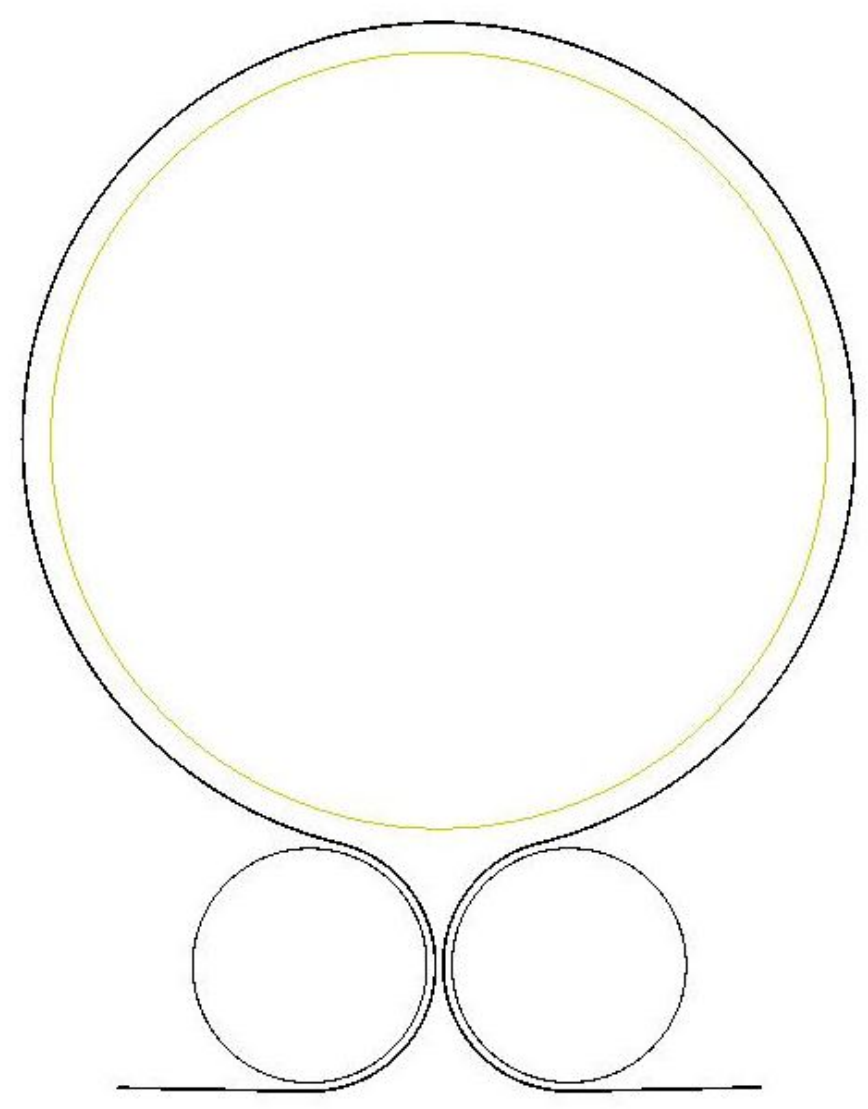

5 

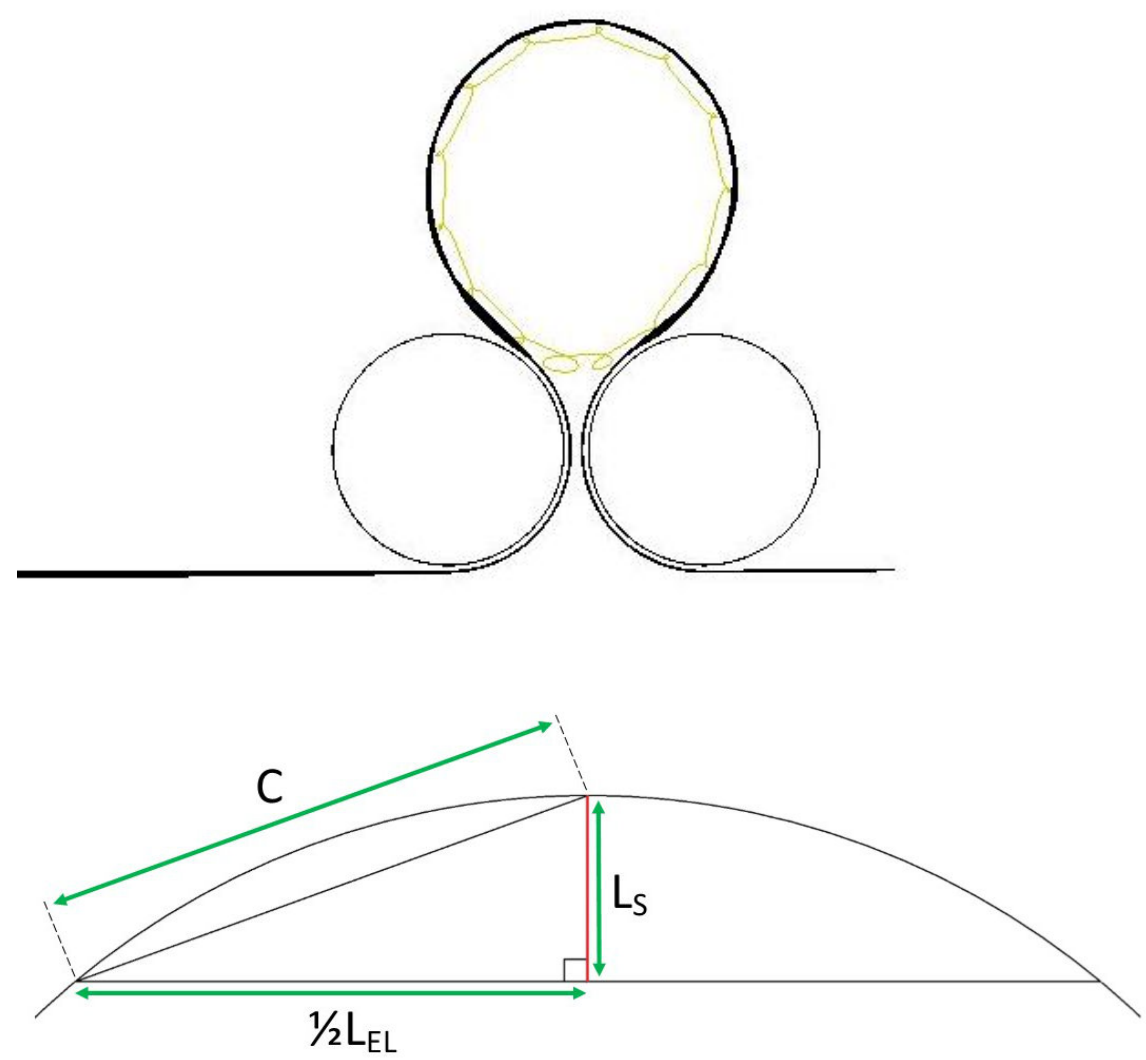


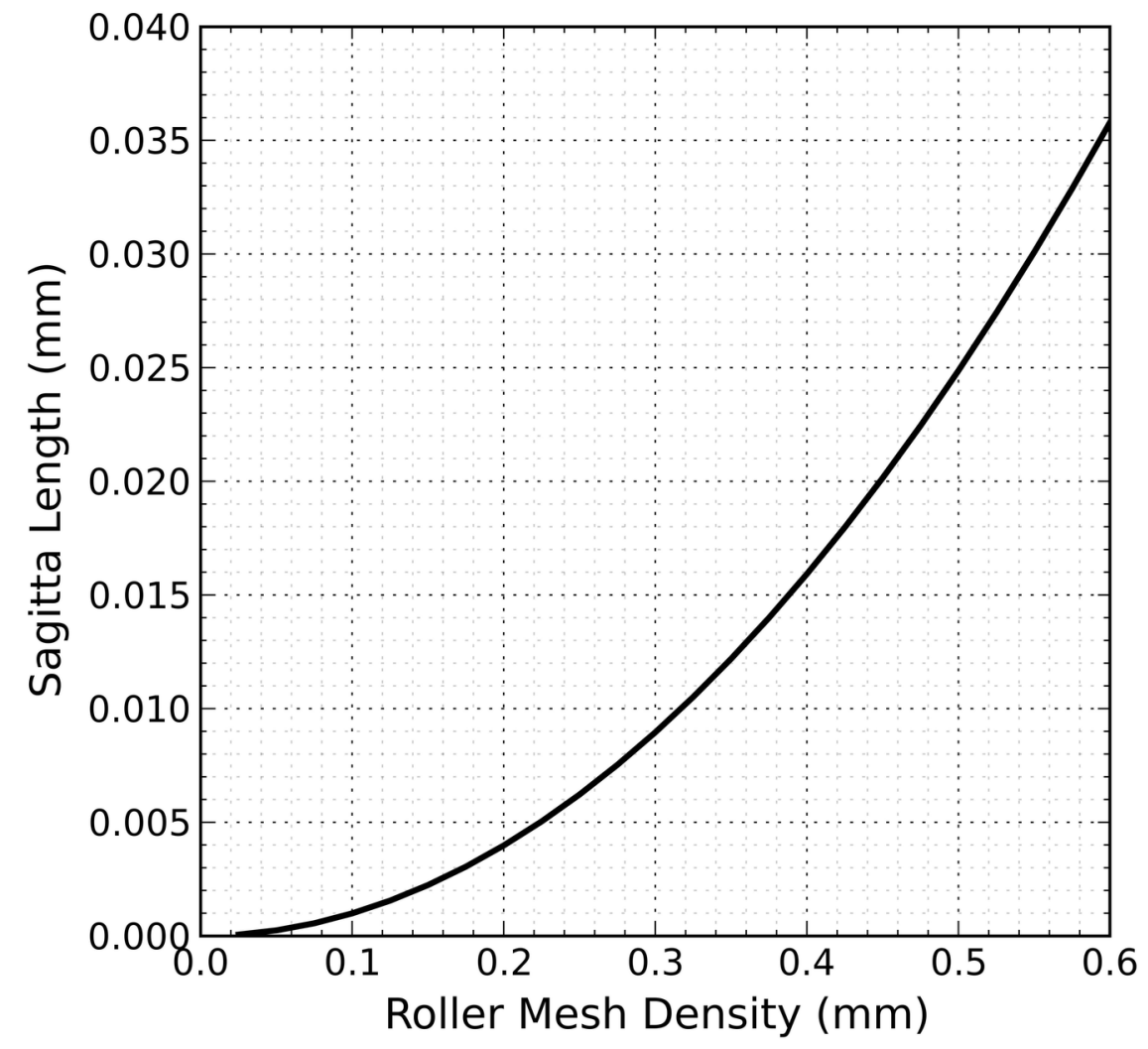




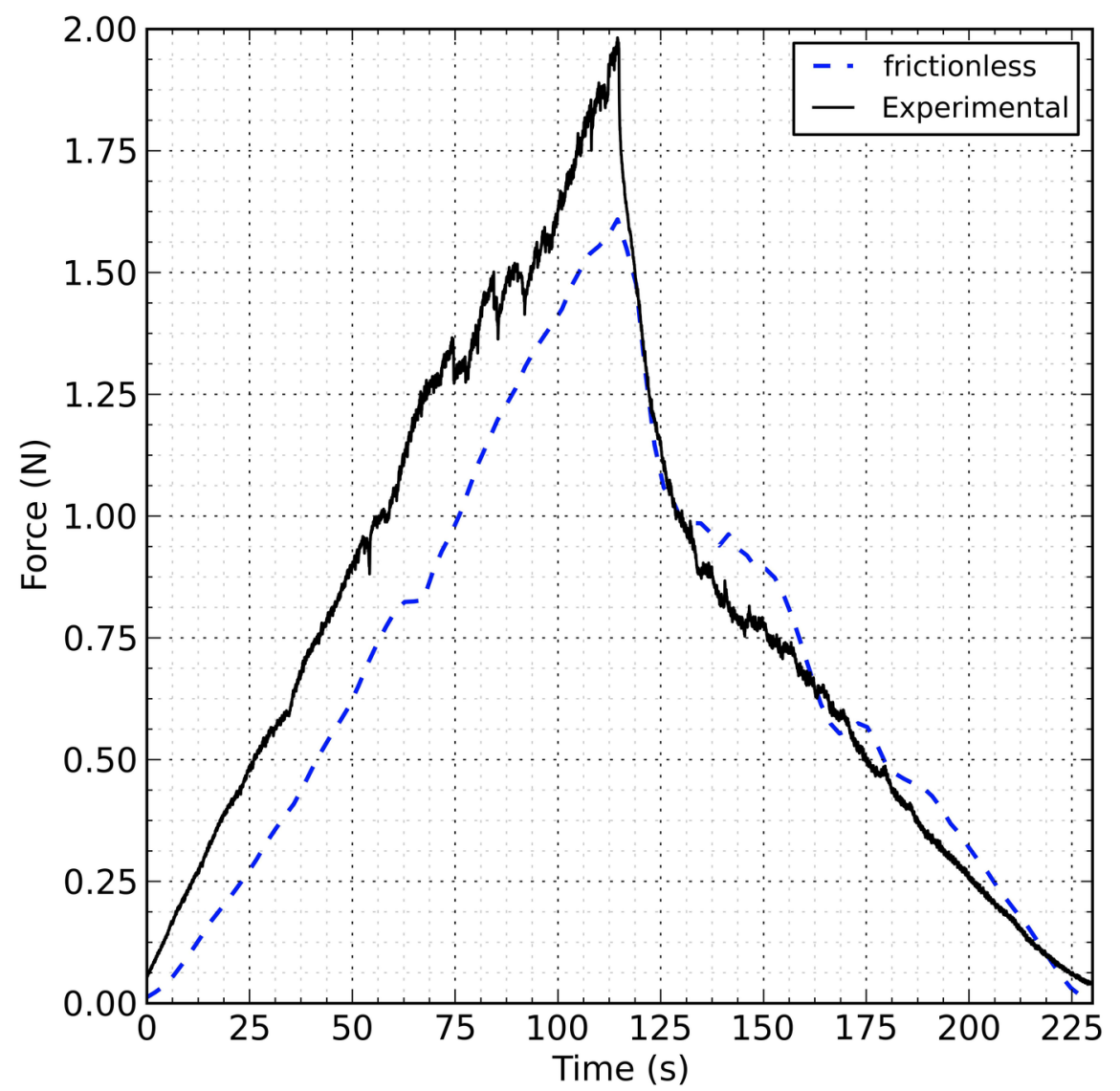




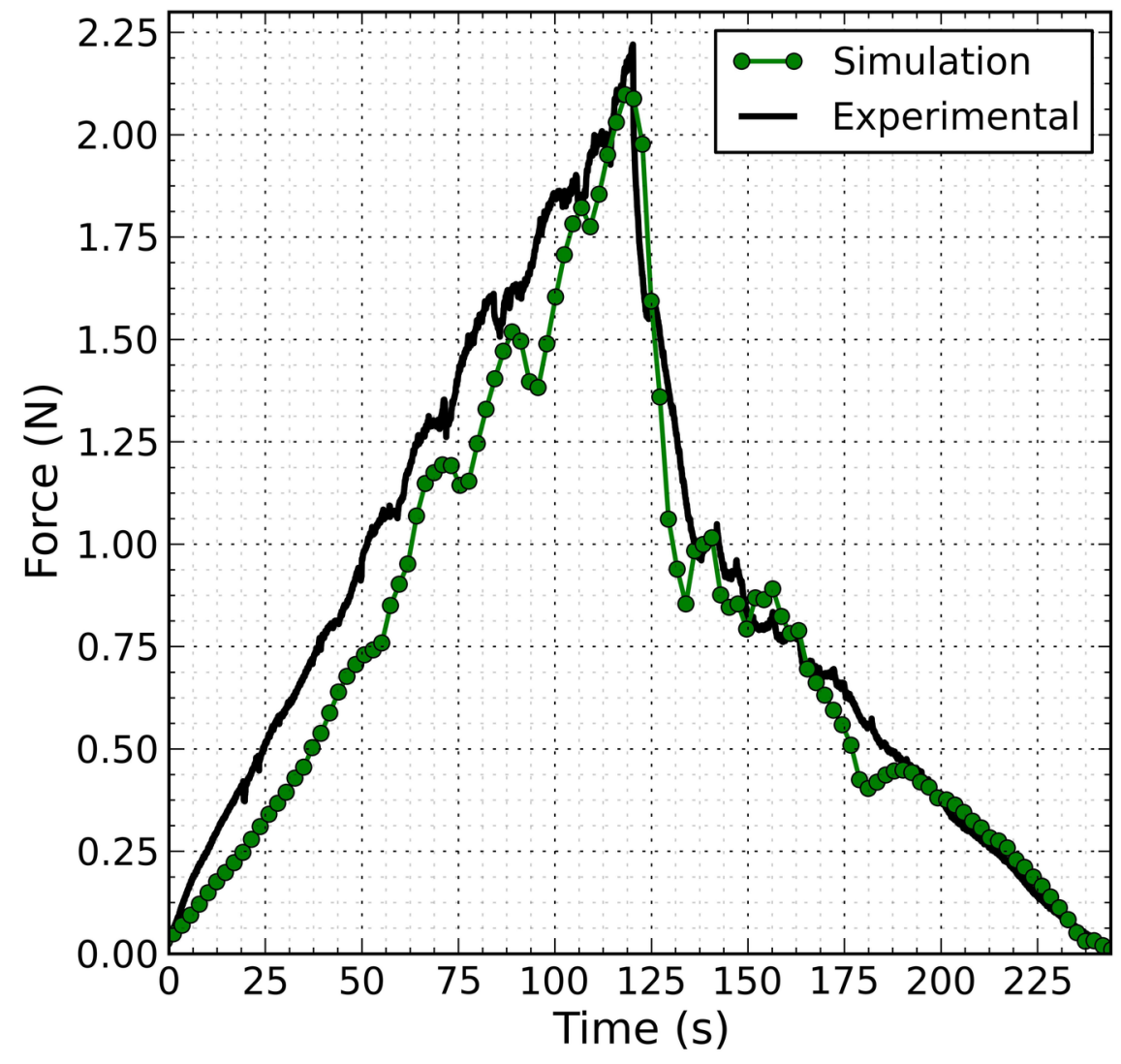



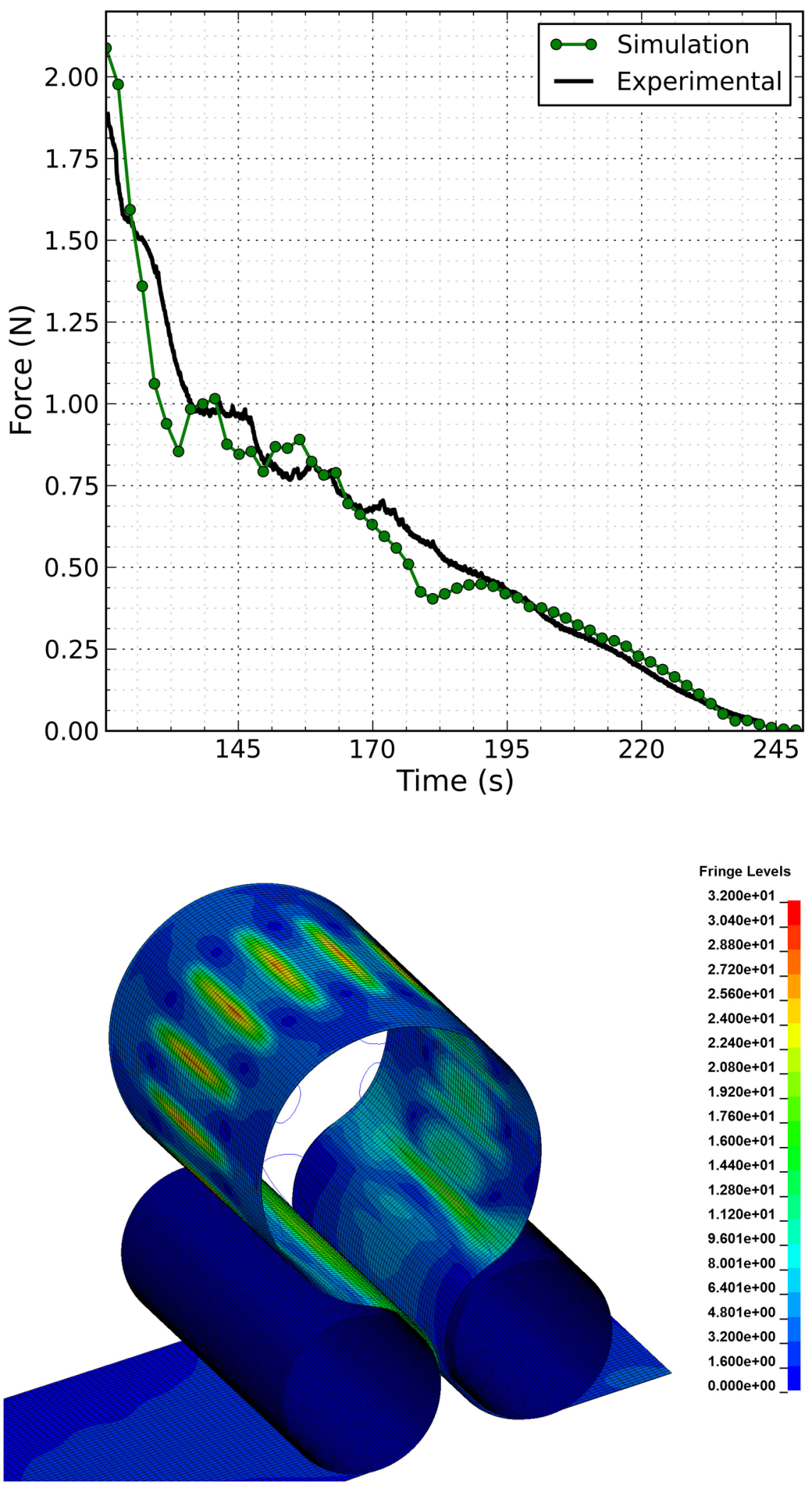

Fringe Levels

$3.200 \mathrm{e}+01$

$3.040 \mathrm{e}+01$

$2.880 \mathrm{e}+01$

$2.720 \mathrm{e}+01$

$2.560 \mathrm{e}+01$

$2.400 \mathrm{e}+01$

$2.240 \mathrm{e}+01$ _

$2.080 \mathrm{e}+01$

$1.920 \mathrm{e}+01$

$1.760 \mathrm{e}+01$

$1.600 \mathrm{e}+01$

$1.440 \mathrm{e}+01$

$1.280 \mathrm{e}+01$

$1.120 \mathrm{e}+01$

$9.601 \mathrm{e}+00$

$8.001 \mathrm{e}+00$

$6.401 \mathrm{e}+00$

$4.801 \mathrm{e}+00$

$3.200 \mathrm{e}+00$

$1.600 \mathrm{e}+00$

$0.000 \mathrm{e}+00$ 


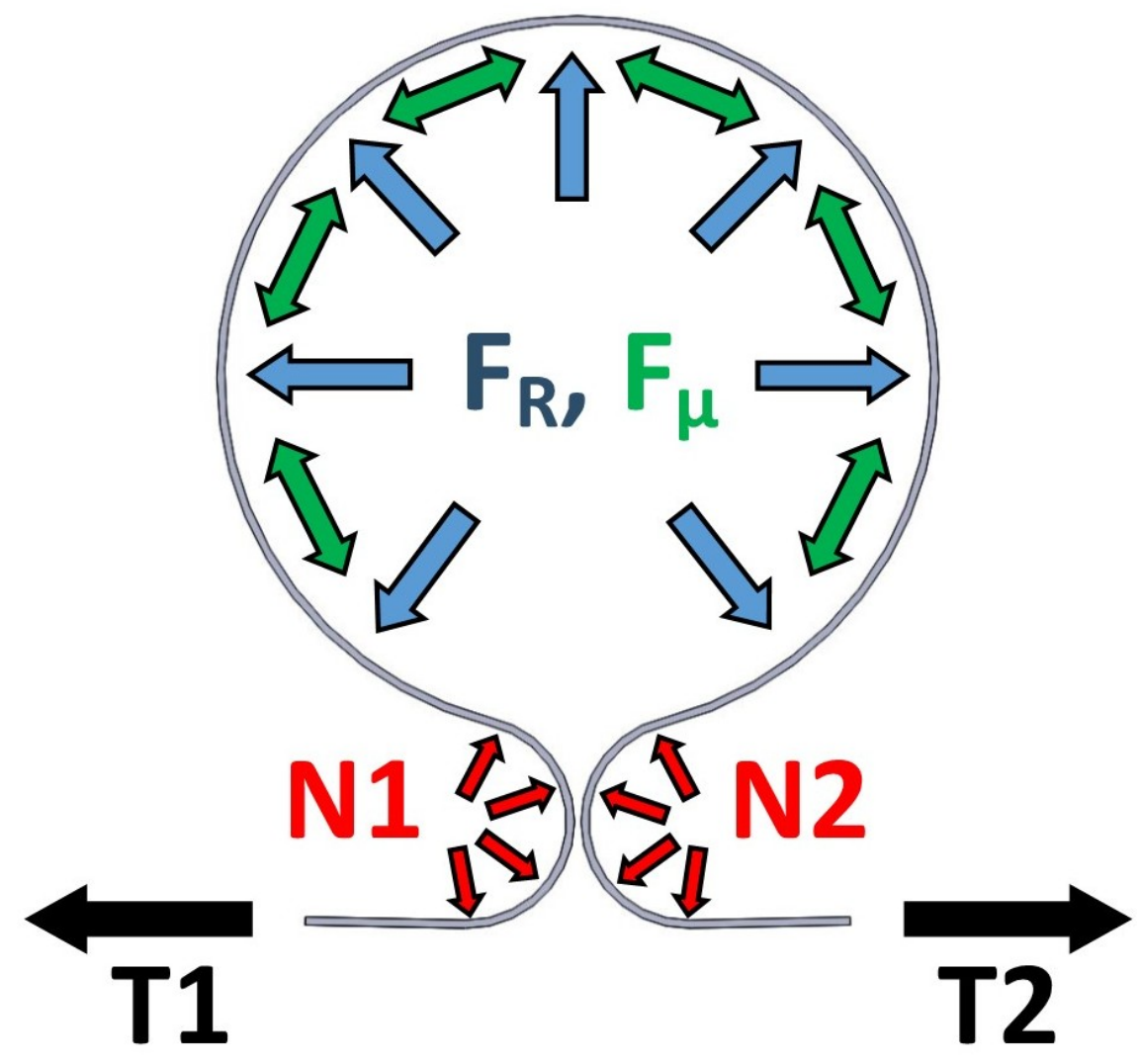



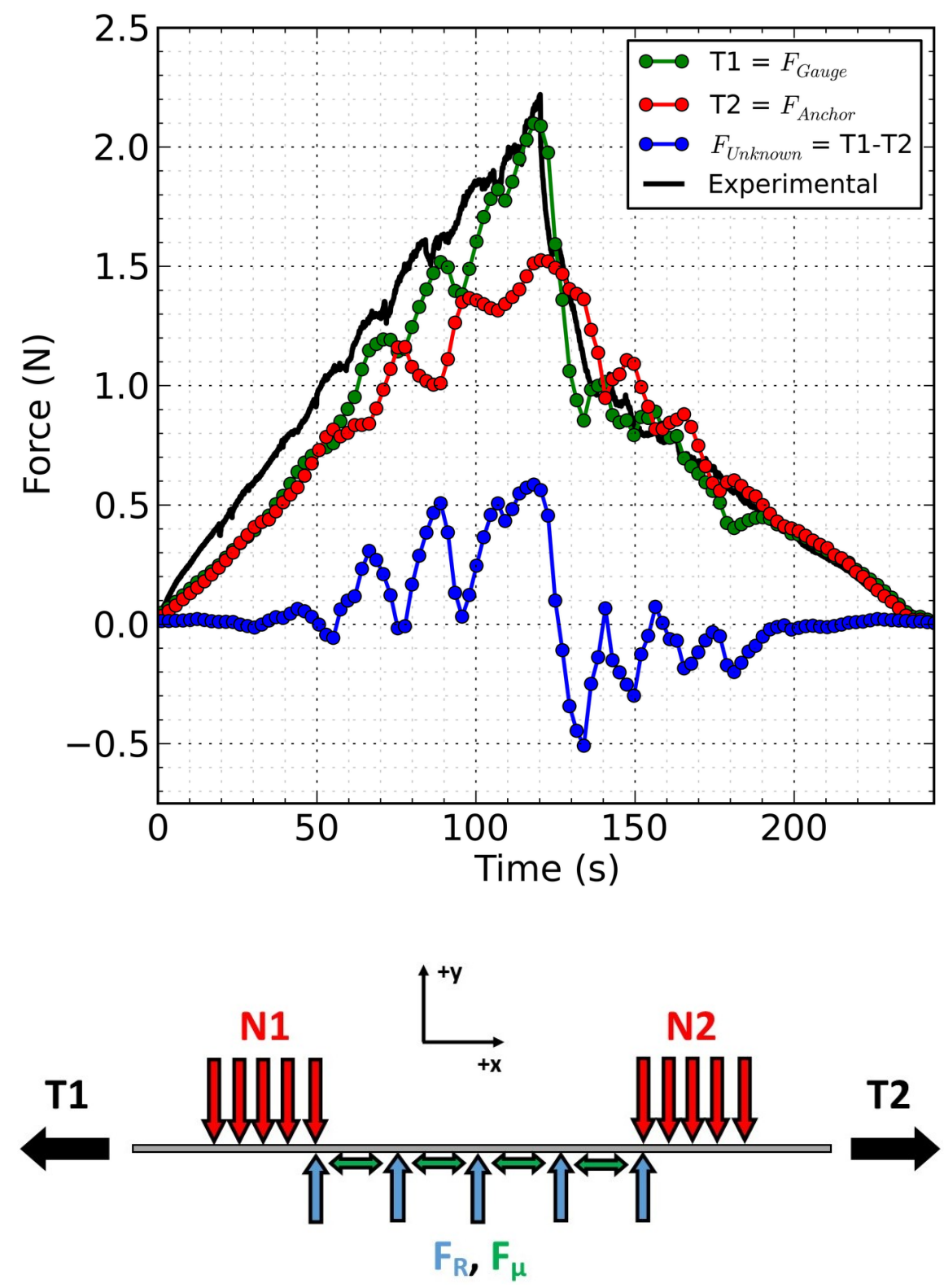

\begin{tabular}{lcc}
\hline \hline Property & Typical Value & Unit \\
\hline Tensile strength, ultimate & 585 & $\mathrm{MPa}$ \\
Tensile strength, yield & 380 & $\mathrm{MPa}$ \\
Young's modulus & 193 & $\mathrm{GPa}$ \\
Density & 0.008 & $\mathrm{~g} / \mathrm{mm}^{3}$ \\
Poisson's ratio & 0.3 & \\
\hline
\end{tabular}




\begin{tabular}{lccc}
\hline \hline Property & Direction & Typical Value & Unit \\
\hline Tensile strength, ultimate & $\mathrm{MD}$ & 196 & $\mathrm{MPa}$ \\
& $\mathrm{TD}$ & 235 & $\mathrm{MPa}$ \\
Young's modulus (tension) & $\mathrm{MD}$ & 4805 & $\mathrm{MPa}$ \\
& $\mathrm{TD}$ & 5001 & $\mathrm{MPa}$ \\
Young's modulus (compression) & & $2726-2834$ & $\mathrm{MPa}$ \\
Density & & 0.00139 & $\mathrm{~g} / \mathrm{mm}^{3}$ \\
Poisson's ratio & & 0.38 & \\
\hline
\end{tabular}

\begin{tabular}{lcccccc}
\hline \hline Coulomb & CPU Time $(\mathrm{s})$ & $F_{\text {Peak }}(\mathrm{N})$ & $r^{2}$ & $S E(\mathrm{~N})$ & MAPE $(\%)$ & MPE $(\%)$ \\
\hline 0.0 & 98638 & 1.61 & 0.967 & 0.072 & 15.20 & -8.29 \\
0.00001 & 92002 & 1.97 & 0.852 & 0.150 & 20.44 & 12.38 \\
0.0001 & 91927 & 2.14 & 0.842 & 0.155 & 20.51 & 11.10 \\
0.0005 & 93533 & 2.04 & 0.860 & 0.146 & 19.70 & 11.61 \\
0.001 & 96466 & 2.10 & 0.914 & 0.114 & 16.01 & 3.76 \\
$\mathbf{0 . 0 0 2 5}$ & $\mathbf{9 8 6 3 8}$ & $\mathbf{2 . 0 4}$ & $\mathbf{0 . 9 4 6}$ & $\mathbf{0 . 0 9 9}$ & $\mathbf{1 1 . 6 9}$ & $\mathbf{1 . 6 9}$ \\
0.005 & 93534 & 2.12 & 0.843 & 0.154 & 20.24 & 11.21 \\
0.0075 & 95597 & 2.04 & 0.865 & 0.143 & 19.64 & 11.73 \\
0.01 & 93155 & 2.15 & 0.811 & 0.169 & 20.48 & 11.28 \\
0.02 & 97391 & 2.13 & 0.934 & 0.100 & 13.56 & 4.55 \\
0.05 & 99542 & 2.11 & 0.919 & 0.111 & 15.92 & 11.52 \\
0.075 & 81722 & 2.40 & 0.776 & 0.184 & 19.20 & 11.53 \\
0.1 & 81794 & 2.47 & 0.750 & 0.195 & 20.74 & 13.42 \\
0.2 & 81794 & 2.62 & 0.502 & 0.275 & 33.96 & 26.70 \\
\hline
\end{tabular}

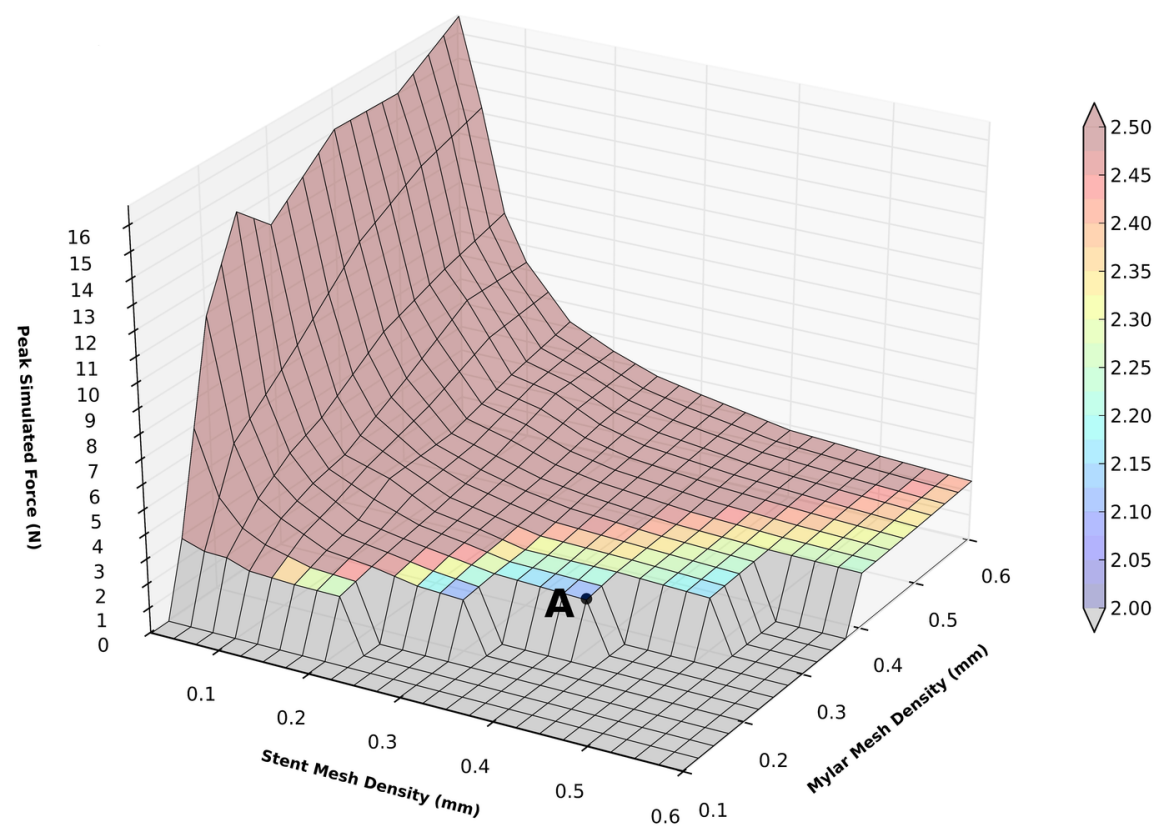




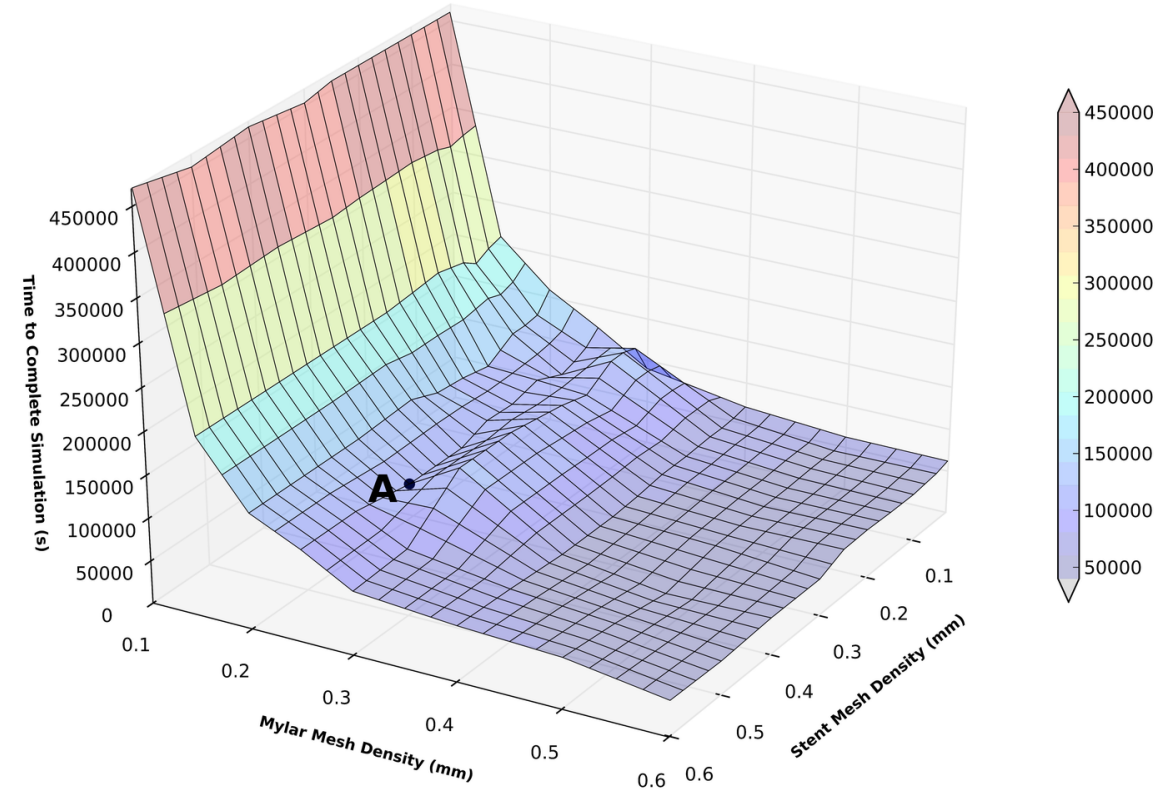

